\title{
Trapped-Mode Resonance Regime of Thin Microwave Electromagnetic Arrays with Two Concentric Rings in Unit Cell
}

\author{
M. N. Kawakatsu and V. A. Dmitriev \\ Department of Electrical Engineering, Federal University of Para, 66075-900 Belem, PA, Brazil \\ Correspondence should be addressed to M. N. Kawakatsu, mnkawakatsu@yahoo.com.br \\ Received 30 September 2010; Accepted 8 April 2011 \\ Academic Editor: Ning Hua Zhu
}

Copyright ( $) 2011$ M. N. Kawakatsu and V. A. Dmitriev. This is an open access article distributed under the Creative Commons Attribution License, which permits unrestricted use, distribution, and reproduction in any medium, provided the original work is properly cited.

We present a theoretical study of reflection and transmission characteristics of a microwave planar array on a thin dielectric substrate with unit cell made of two concentric rings. This array possesses high quality factor transmission resonance with polarization insensitivity for normally incident plane wave. This resonance is defined by the trapped-mode regime. We show that for oblique incidence, there are some differences in characteristics of the array and a small change in quality factor of the trapped-mode resonance.

\section{Introduction}

Planar arrays consisting of a single resonant element (patch or aperture) in the unit cell, often called frequency selective surfaces (FSS) are traditionally used in the microwave and optical regions as filters, in the design of antennas and antenna radomes, and in the far-infrared region, as polarizers, beam splitters, and laser cavity mirrors [1]. An intrinsic feature of these FSSs is a resonance with relatively low quality $(Q)$ factor. The low value of quality factor is explained by the fact that a thin open structure cannot have inner resonating volume and the resonating inclusions are strongly coupled with the free space. However, for some applications, a thin surface with high $Q$-factor resonance is desired.

There exists a method to produce very thin structures possessing high $Q$-factor frequency resonances using the socalled trapped-mode resonance regime [2]. The trappedmode can be excited in two element arrays when the resonance frequencies of the elements are approximately equal. This mode is characterized by a sharp resonance and strong current intensities which are of opposite directions in the two elements. Due to the almost complete cancelation of the dipole moments, the fields radiated by this current distribution are very weak and also the coupling of this mode with free space is weak. As a consequence, the radiation loss of the trapped-mode resonance is dramatically reduced resulting in a high Q-factor compared with the usual resonance of array with one element in unit cell.

Some applications of these planar metamaterials are described in $[3,4]$. In [3], the authors show that these arrays can be used in a spaser (surface plasmon amplification by stimulated emission of radiation) to produce a spatially and temporally coherent electromagnetic radiation source designated as laser spaser. In [4], an approach for sensing a small amount of chemical and biochemical material using an array constituted by asymmetrically double split ring elements is discussed.

In [5], the authors presented a detailed study of the trapped-mode in an array of two concentric rings and suggested a new array which is formed by an outer circular and inner corrugated rings in order to achieve a trappedmode regime with higher $Q$-factor. The structures were parametrically optimized. But in paper [5], only normal incidence of electromagnetic waves was studied which limits application of the theory. The contribution of this work is a theoretical study of the array made of two concentric rings in unit cell for an arbitrary angle of incidence.

\section{Description of the Array}

The periodic array analyzed in this work and its unit cell with two concentric rings are shown in Figure 1. In Figure 1(a), 


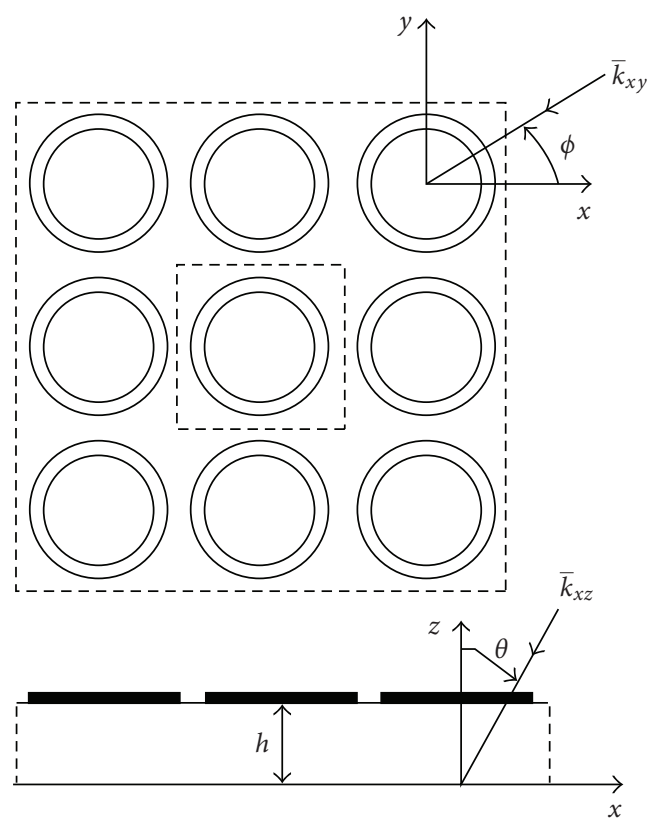

(a)

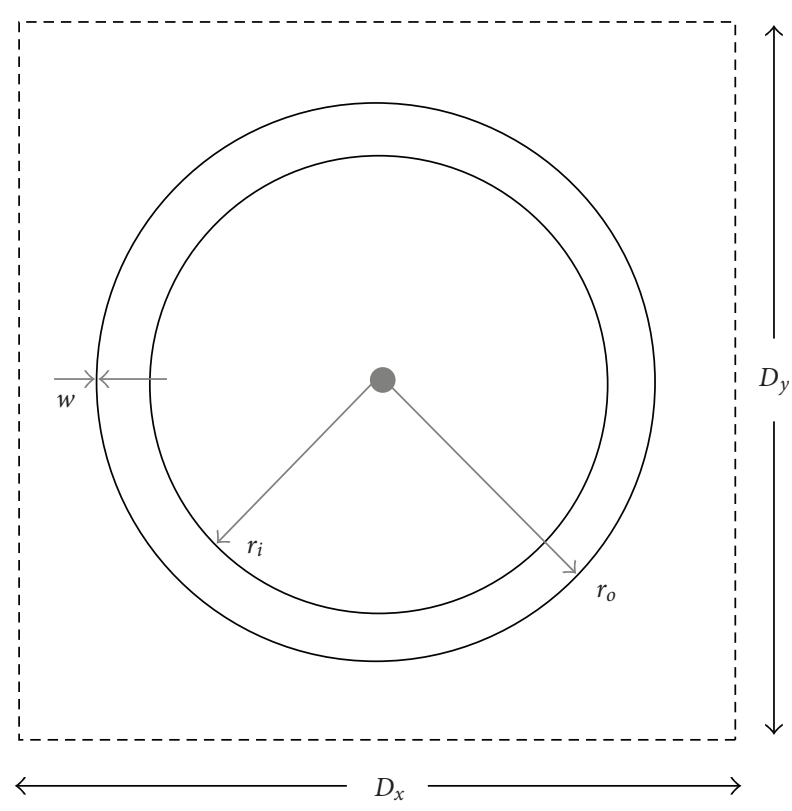

(b)

FIgURE 1: Analyzed array with the reference coordinate system (a), a unit cell of the array (b).

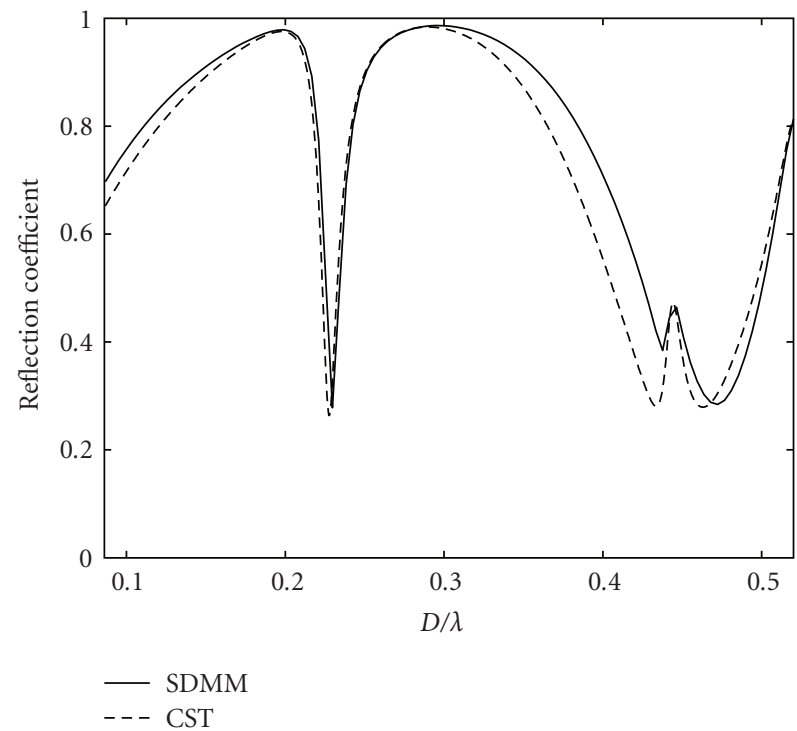

(a)

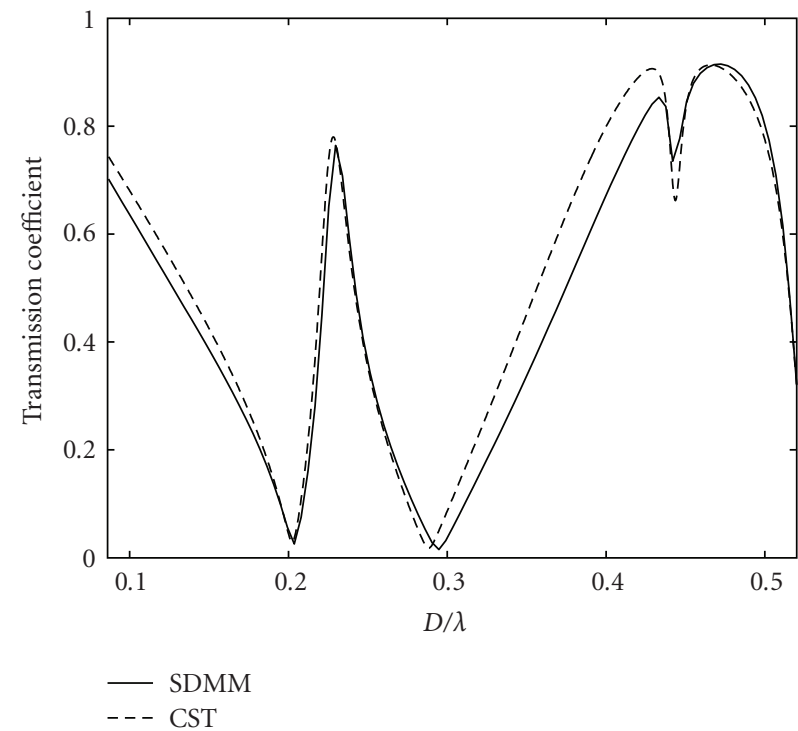

(b)

Figure 2: Reflection (a) and transmission (b) coefficients for horizontally polarized incident plane wave, $\phi=45^{\circ}, \theta=45^{\circ}, \varepsilon=4.5-i 0.1$.

the Cartesian coordinate system is shown as well. To describe the direction of the propagation vector of the incident plane wave, we use the spherical angular coordinates: the azimuthal angle $\phi$ and the elevation angle $\theta$. In Figure $1(\mathrm{a}), \bar{k}_{x y}$ and $\bar{k}_{x z}$ are components of the wave vector of the incident wave in the planes $x y$ and $x z$, respectively.

The unit cell has the dimensions $D_{x}$ and $D_{y}$ which are the periods of the array in $x$ and $y$ directions. The dimensions of the unit cell considered in this work are $D_{x}=D_{y}=$ $13 \mathrm{~mm}$; the middle radius of the outer and inner rings are, respectively $r_{0}=6$ and $r=5 \mathrm{~mm}$. Both rings are made of a metallic strip of width $w=0.2 \mathrm{~mm}$ and considered to be perfectly conducting ones. The substrate is of thickness $h=1.6 \mathrm{~mm}$ with the dielectric constant $\varepsilon=\varepsilon^{\prime}-i \varepsilon^{\prime \prime}$.

\section{Numerical Method of Analysis}

To analyze this structure, we developed a computer program based on the Spectral Domain Moment Method (SDMM) which consists in solving by the method of moments 


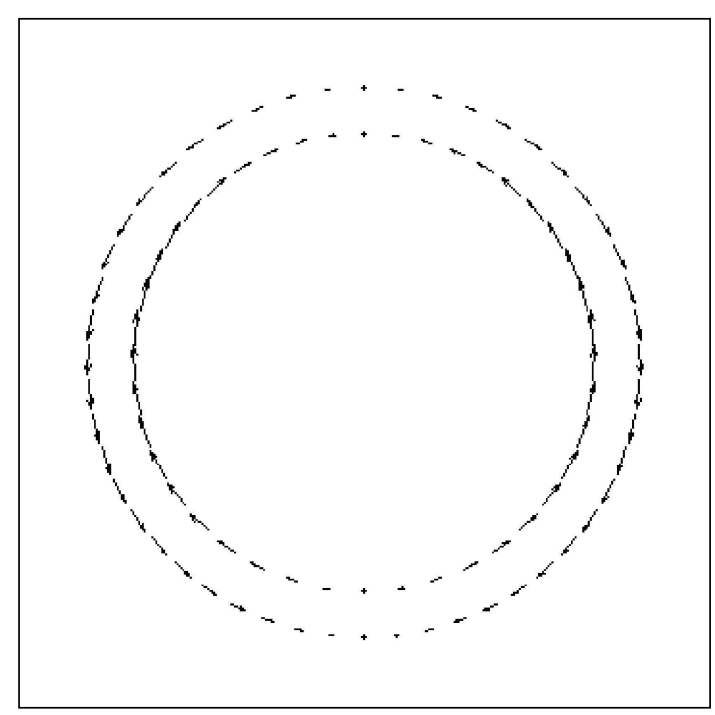

(a)

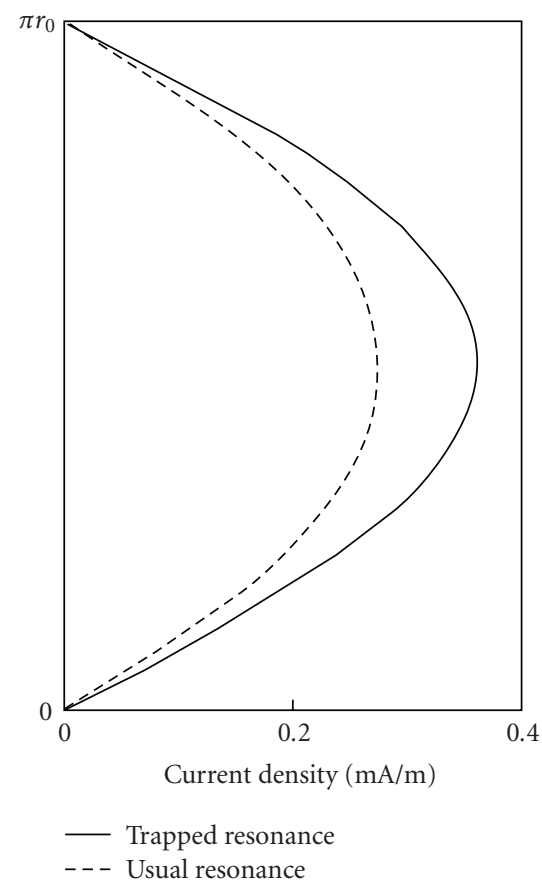

(b)

FIGURE 3: Current distribution on the rings for y-polarized normally incident plane wave. In (a), the normalized current distributions on the two rings are shown, and in (b) the absolute values of current density on one half of the outer ring at trapped resonance and at usual resonance are presented.

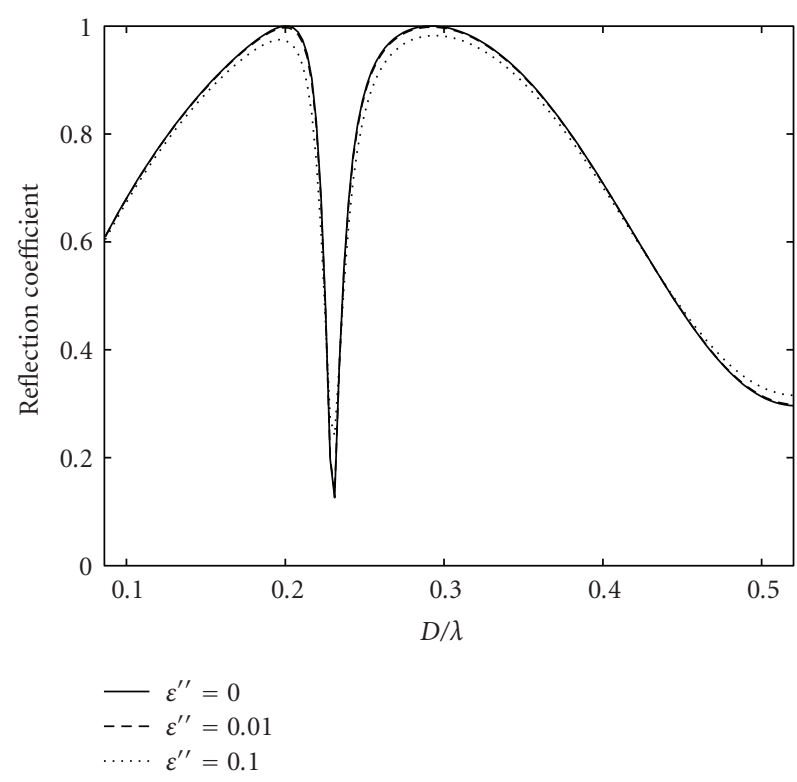

(a)

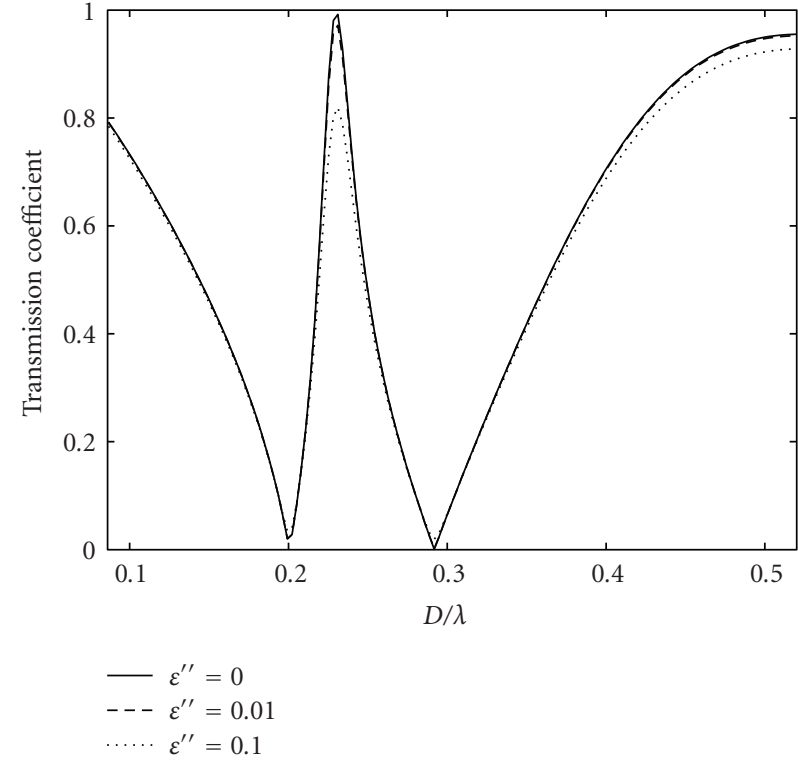

(b)

Figure 4: Reflection (a) and transmission (b) coefficients for normally incident plane wave, $\varepsilon^{\prime}=4.5$, different $\varepsilon^{\prime \prime}$.

the electric field integral equation in the spectral domain. In this method, the periodic boundary conditions are taken into account by transforming the continuous spectral domain variables into harmonics that are multiples of the periodicities of the array. The substrate is modeled by the Green's function which is obtained by applying the boundary conditions on the top and the bottom interfaces. This method is described in [1]. For validation of our program, we used the commercial software Computer Simulation Technology (CST) which is based on the finite integration technique [6]. 


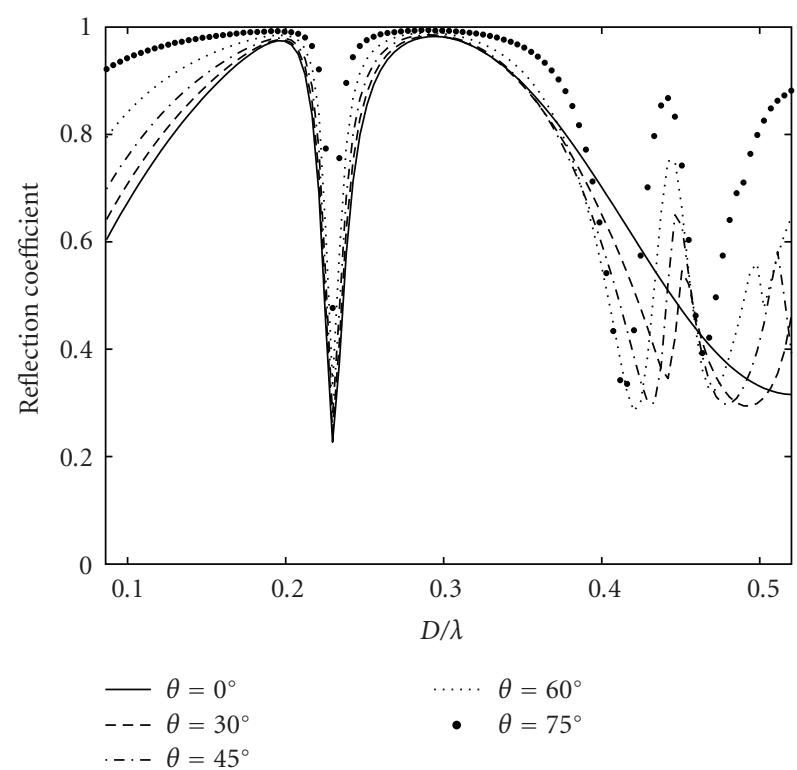

(a)

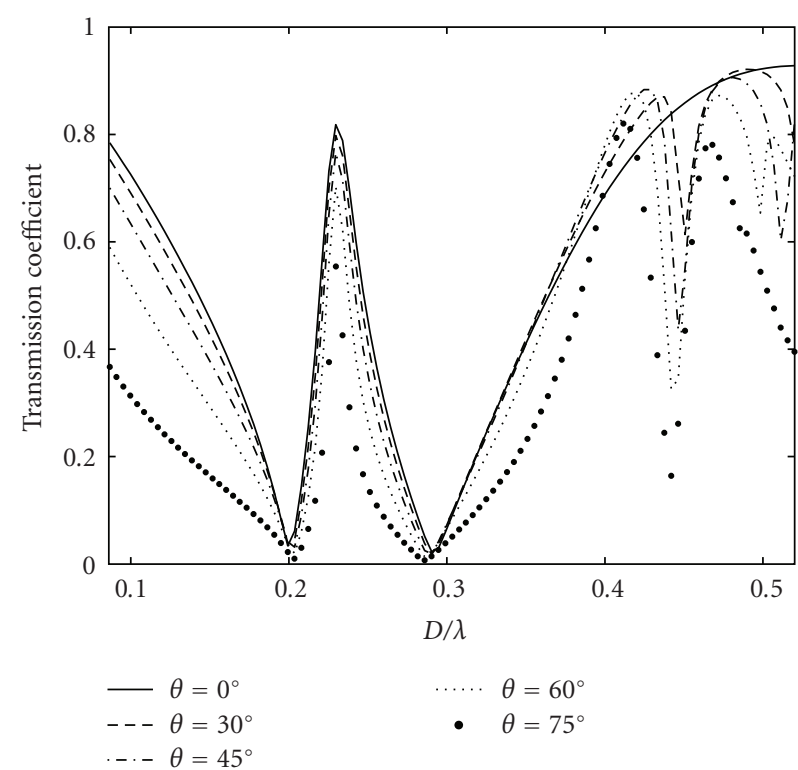

(b)

FIGURE 5: Reflection (a) and transmission (b) coefficients for horizontally polarized incident plane wave, $\phi=0^{\circ}$, different $\theta$.

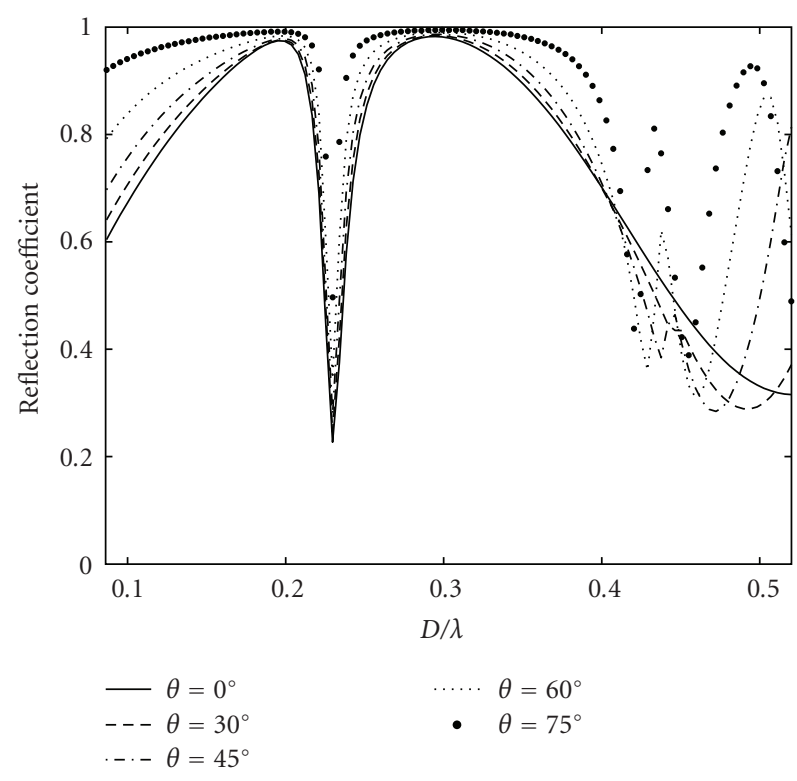

(a)

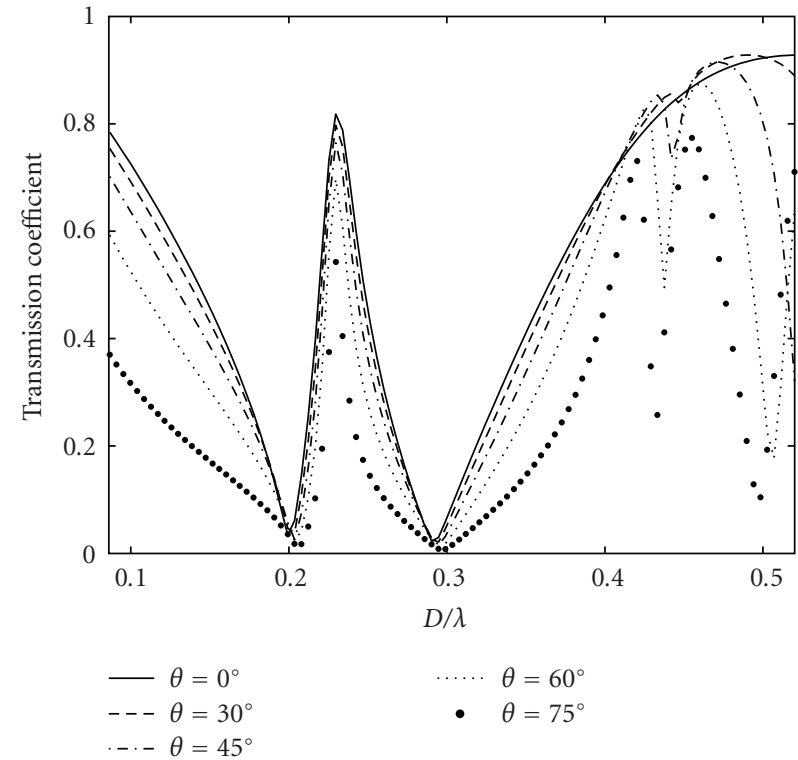

(b)

FIGURE 6: Reflection (a) and transmission (b) coefficients for horizontally polarized incident plane wave, $\phi=45^{\circ}$, different $\theta$.

The structure is excited by a plane wave incident from the half space $z>h$ above the metal array (Figure 1(a)). In Figures 2(a) and 2(b), we show the reflection and transmission coefficients (defined in terms of electric field) for horizontally polarized incident plane wave, $\phi=45^{\circ}, \theta=45^{\circ}$, $\varepsilon=4.5-i 0.1$. The curves in these figures were calculated using our SDMM and CST programs.

We see in Figure 2 that the reflection and transmission coefficients calculated by two programs are in a good agreement. The numerical differences between the results may be caused by the fact that in the SDMM, the dielectric slab is taken into account analytically by the Green's function while in the CST it is modeled numerically through small finite volumes of constant equivalent current density. To our opinion, the first method is more adequate for solving the problem. For that reason, we will show in the following only the results obtained by the SDMM.

\section{Brief Description of Trapped-Mode Regime}

To illustrate the trapped-mode regime, we show in Figure 3(a) the calculated current distributions on the rings of a unit cell at the trapped-mode resonance frequency for 


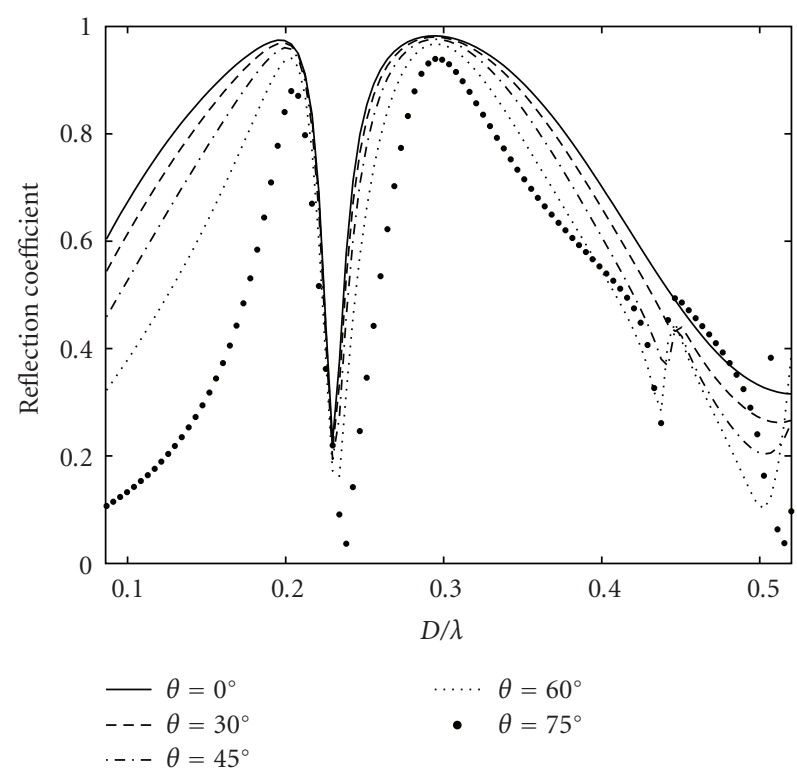

(a)

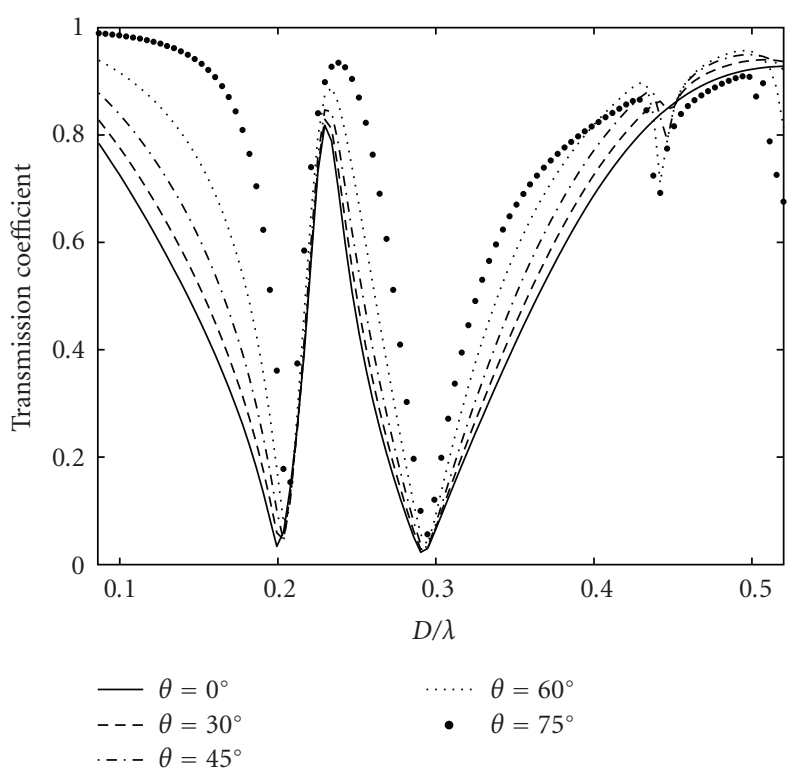

(b)

Figure 7: Reflection (a) and transmission (b) coefficients for vertically polarized incident plane wave, $\phi=0^{\circ}$, different $\theta$.

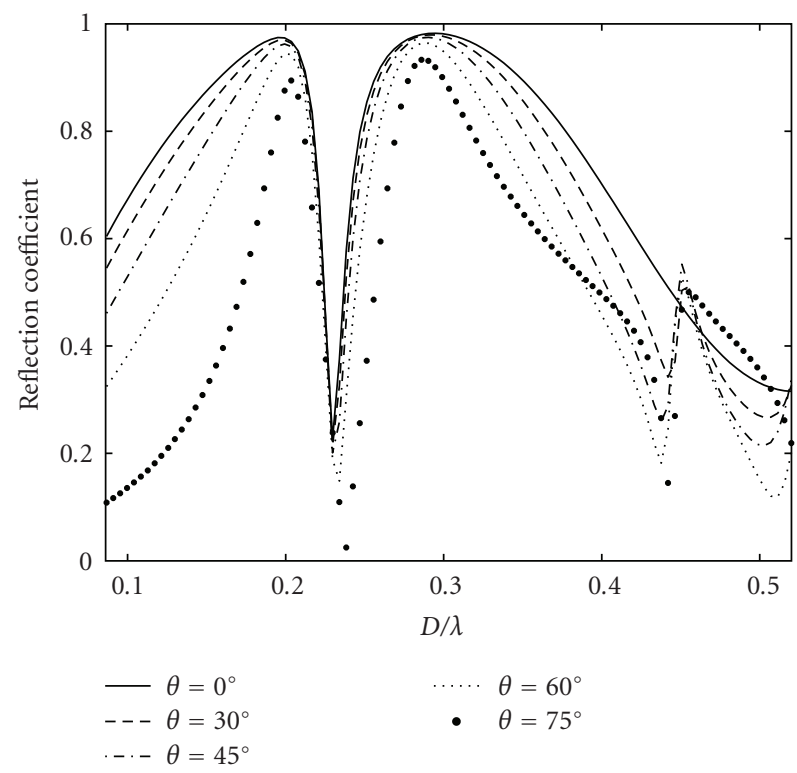

(a)

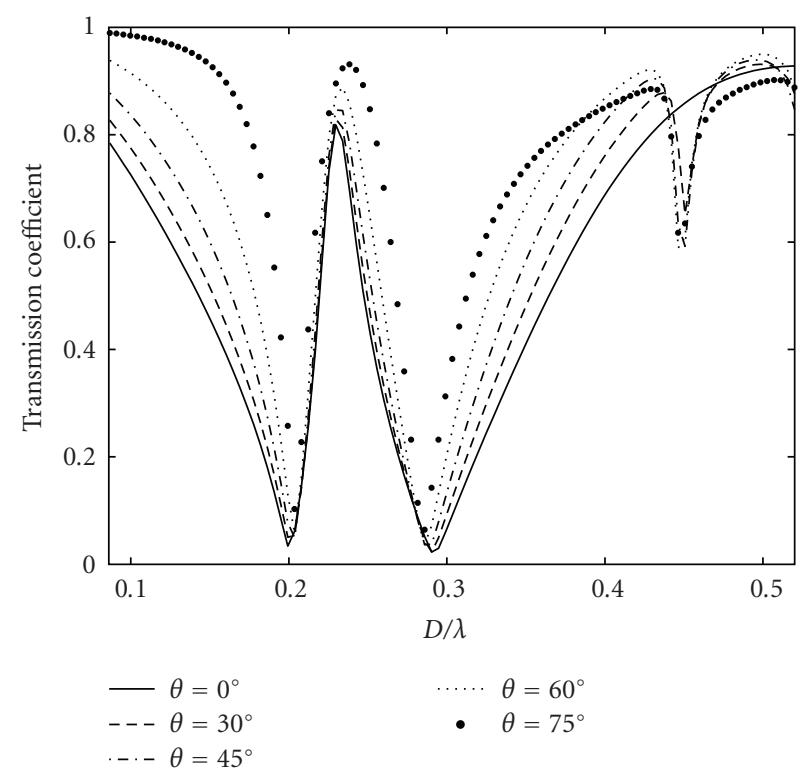

(b)

Figure 8: Reflection (a) and transmission (b) coefficients for vertically polarized incident plane wave, $\phi=45^{\circ}$, different $\theta$.

the case of normal incidence of the y-polarized plane wave. As it was explained before, the trapped-mode corresponds to a high asymmetric current mode which is weakly coupled to free space. In Figure 3(b), the current density along one half of the outer ring at trapped-mode resonance and at usual resonance of the outer ring is shown.

\section{Results}

5.1. Influence of Dielectric Losses on the Trapped-Mode. To illustrate the influence of the dielectric loss on the trapped- mode regime, we show in Figure 4 the structure responses for $\varepsilon^{\prime}=4.5$ and different values of $\varepsilon^{\prime \prime}$ for the case of normally incident wave. The normalized resonance frequency of the trapped-mode is $D / \lambda \approx 0.23$.

For the dielectric substrate without losses, the transmission coefficient at the trapped-mode resonance is almost equal to one. We can see also from Figure 4(b) that for the dielectric with $\varepsilon^{\prime \prime}=0.1$ the transparence of the array at the trapped-mode resonance frequency is reduced to the value 0.8 due to the dielectric loss. In the following analysis, we 


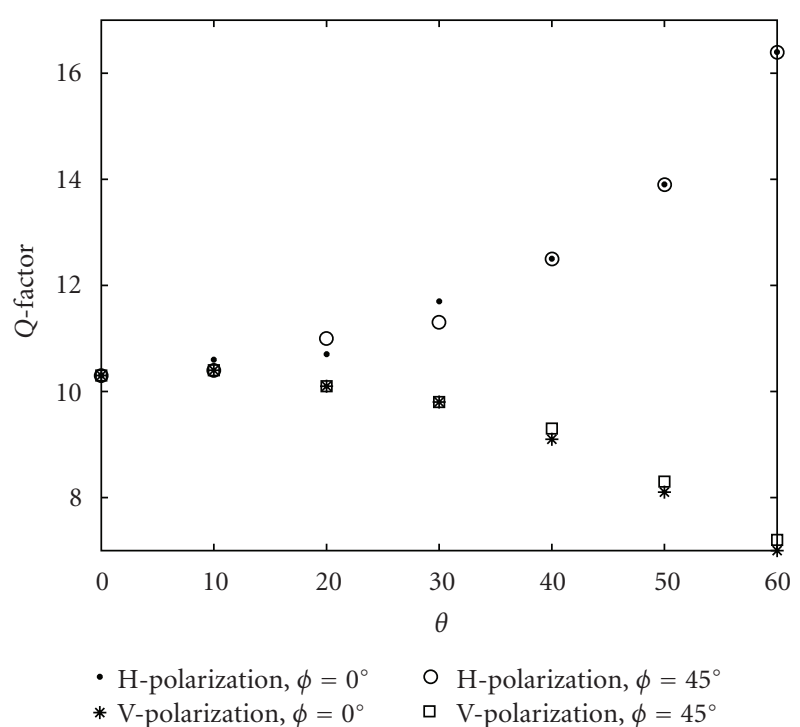

FIGURE 9: Quality factor Q for horizontally $(\mathrm{H})$ and vertically (V) polarized incident plane wave as a function of $\theta$ for $\phi=0^{\circ}$ and $\phi=45^{\circ}$.

consider the dielectric with permittivity $\varepsilon=4.5-i 0.1$ in order to make an evaluation of the structure responses in the presence of dielectric losses.

5.2. Horizontal Polarization. Below, we show the structure responses for horizontally polarized incident wave and four values of $\theta\left(\theta=0^{\circ}, 30^{\circ}, 45^{\circ}\right.$ and $\left.60^{\circ}\right)$, in Figure 5 for $\phi=0^{\circ}$ and in Figure 6, for $\phi=45^{\circ}$.

We see in Figures 5 and 6 that the resonance frequency $D / \lambda \approx 0.23$ of the trapped-mode for horizontally polarized incident wave practically does not depend on the angle of incidence (at least for the calculated angles up to $\theta=60^{\circ}$ ) for both $\phi=0^{\circ}$ and $\phi=45^{\circ}$, azimuthal angles. When the angle of incidence is increased the bandwidth of the trappedmode is reduced as well as the transparence at the resonance frequency. Also, increasing the angle $\theta$, sharp peaks in the reflection coefficient and transmission coefficient deeps appear on the frequency range of $D / \lambda \approx 0.4-0.5$.

5.3. Vertical Polarization. In Figure 7, we show the structure responses for the vertically polarized incident plane wave, different values of angle $\theta, \phi=0^{\circ}$, and in Figure 8, for $\phi=45^{\circ}$. We observe a different behavior of the trappedmode in comparison with the case of horizontal polarization. First of all, increasing $\theta$ the resonance frequency is slightly shifted to the higher ones. Secondly, the bandwidth and the transparence at the trapped-mode resonance are slightly increased. Another peculiarity of this case is that the peaks in the reflection coefficient and transmission coefficient deeps appear at higher angles of incidence $\theta$, and they are not so pronounced as for the case of horizontal polarization.

5.4. Quality Factor. We define the quality factor $Q$ as the ratio between the resonance frequency and the full bandwidth at level $-3 \mathrm{~dB}$ from the maximum of the transmission coefficient. For horizontally $(\mathrm{H})$ and vertically $(\mathrm{V})$ polarized incident plane wave, we show in Figure 9 the $Q$-factor as a function of $\theta$ for $\phi=0^{\circ}$ and $\phi=45^{\circ}$. One of the conclusions following from this figure and from the results presented above is that the trapped-mode resonance of the array is practically not sensitive to the azimuthal angle $\phi$ because the period of the array is small in comparison with the wavelength, $D / \lambda \approx 0.23$.

\section{Conclusion}

A theoretical study of reflection and transmission characteristics of the array with a unit cell formed by two concentric rings supported by a dielectric slab was presented. The structure was analyzed for normal and oblique incidence of electromagnetic plane waves. It was shown that for horizontal polarization of incident wave the resonance frequency does not depend on the angle of incidence but for vertical polarization there is a small shift of the resonance frequency. Also, the trapped-mode bandwidth and transparency at the resonance frequency are slightly reduced when the angle of incidence is increased for the case of horizontal polarization, and they are increased for the case of vertical polarization.

\section{Acknowledgment}

The authors would like to acknowledge the financial support of the Brazilian agencies CAPES and CNPq.

\section{References}

[1] R. Mittra, C. H. Chan, and T. Cwik, "Techniques for analyzing frequency selective surfaces-a review," Proceedings of the IEEE, vol. 76, no. 12, pp. 1593-1615, 1988.

[2] S. Prosvirnin and S. Zouhdi, "Resonances of closed modes in thin arrays of complex particles," in Proceedings of the International Conference on Electromagnetics of Complex Media and Metamaterials, pp. 281-290, 2003.

[3] N. I. Zheludev, S. L. Prosvirnin, N. Papasimakis, and V. A. Fedotov, "Lasing spaser," Nature Photonics, vol. 2, no. 6, pp. 351-354, 2008.

[4] C. Debus and P. H. Bolivar, "Frequency selective surfaces for high sensitivity terahertz sensing," Applied Physics Letters, vol. 91, no. 18, Article ID 184102, 2007.

[5] M. N. Kawakatsu, V. A. Dmitriev, and S. L. Prosvirnin, "Microwave frequency selective surfaces with high Q-factor resonance and polarization insensitivity," Journal of Electromagnetic Waves and Applications, vol. 24, no. 2-3, pp. 261-270, 2010.

[6] http://www.cst.com/. 

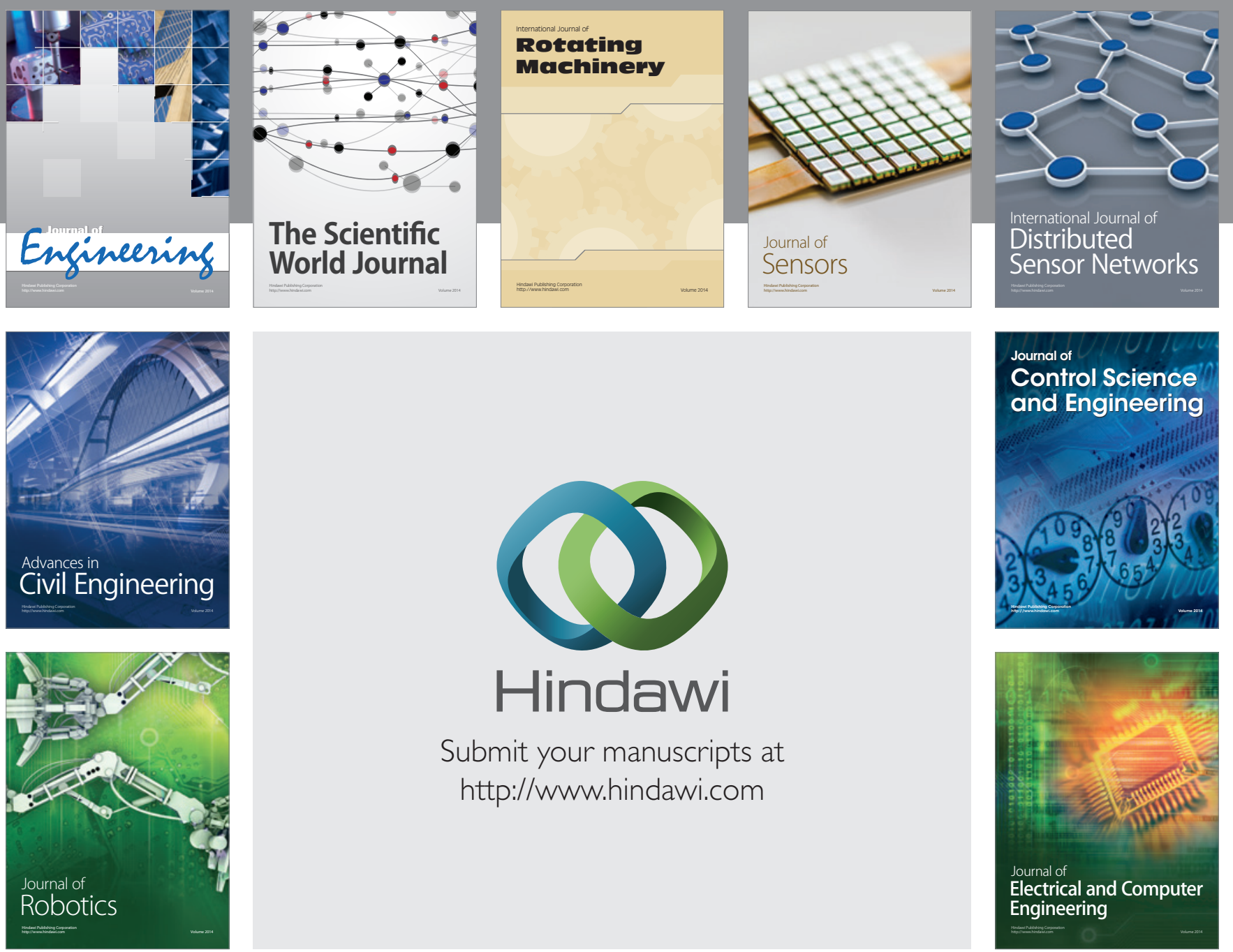

Submit your manuscripts at

http://www.hindawi.com
